\title{
Design of automatic urine collection system for medical system applications
}

\author{
Li Cheng $^{\mathrm{a}, \mathrm{b}}$, Chenru Hao ${ }^{\mathrm{a}, \mathrm{b}, *}$, Yanpeng Wang ${ }^{\mathrm{c}}$, Jingjing Zhang ${ }^{\mathrm{a}}$, Yunlong Wen ${ }^{\mathrm{d}}$, Ziqiang Chi ${ }^{\mathrm{a}}$, \\ Xiuyuan $\mathrm{Li}^{\mathrm{e}}$, Haibo Yang ${ }^{\mathrm{a}}$, Yanru $\mathrm{Wu}^{\mathrm{a}}$, Lisha Guo ${ }^{\mathrm{a}}$ and Ruibin $\mathrm{Zhao}^{\mathrm{a}}$ \\ ${ }^{a}$ Department of Medical Physics, Hebei Medical University, Shijiazhuang, Hebei, China \\ ${ }^{\mathrm{b}}$ Key Labarotory of Medical Imaging Research and Application of Hebei Province, Shijiazhuang, Hebei, \\ China \\ ${ }^{\mathrm{c}}$ Department of Electrical and Computer Engineering, Laval University, Quebec, Canada \\ ${ }^{\mathrm{d}}$ Shijiazhuang Yuxun Electronics Co., Ltd, Shijiazhuang, Hebei, China \\ ${ }^{\mathrm{e}}$ Department of Foreign Languages, Hebei Medical University, Shijiazhuang, Hebei, China
}

\begin{abstract}
.
BACKGROUND: The results of urine tests are often affected by improper midstream urine collection time, urine spilling, and urine pollution, all of which can lead to an increase in the test error.

OBJECTIVE: To solve this problem, aiming at improving the toilet environment at the hospitals and public physical examination centers, this paper designs an automatic urine collection system. It can automatically adjust the position of the urine cup with an infrared remote controller, or manually, adjust the position of the urine cup in special situations according to the needs of the user. It also has an alarm function.

METHODS: The overall size and shape are designed based on the squatting pan, suitable for disposable plastic urine cups of different shapes and sizes. It can realize the automatic collection of midstream urine, manual collection in exceptional cases, emergency stops, and rescue calls.

RESULTS: Through the trial survey, there was a significant difference between the statistical results of using the device and not using the device ( $t=13.937, P=0.000) .96 \%$ of the subjects thought that the design of the system was reasonable, $22 \%$ thought that it was inconvenient to use, and $91.7 \%$ of the medical staff thought that the system met the sampling requirements. CONCLUSIONS: Therefore, the trial evaluation is satisfactory, and the proposed collection system is suitable for use in hospitals at all levels and public health examination centers with a large amount of inspection.
\end{abstract}

Keywords: Automatic collection system, medical physical examination, single-chip microcomputer, urine test

\section{Introduction}

Urine examination is a kind of medical diagnostic method. Through the test of urine, doctors can obtain relevant information about the kidney and urinary system. Urine examination is a routine examination and is also the most common examination in nephrology. The results of urine tests are significant for clinical diagnosis, curative effect, and prognosis, providing valuable evidence across urology, antenatal health, diabetes, and increasingly certain cancers [1-3].

\footnotetext{
${ }^{*}$ Corresponding author: Chenru Hao, Department of Medical Physics, Hebei Medical University, No. 48 Donggang Road, Shijiazhuang, Hebei, China. E-mail: 793699829@qq.com.
}

0928-7329 (c) 2022 - The authors. Published by IOS Press. This is an Open Access article distributed under the terms of the Creative Commons Attribution-NonCommercial License (CC BY-NC 4.0). 
Urine tests are usually performed in hospitals or public health centers. The first step of the urine test is urine collection. At present, the primary collection method in urine test is to distribute disposable plastic urine cups by medical personnel, and the subjects take samples alone or accompanied by their family members. Because the examinees mainly control this process, there are often some factors, such as improper midstream urine collection time, urine spilling, and urine pollution, which lead to the increase of the test error. Besides, the plastic sampling cup is easy to bend and deform, which is not easy to operate, especially for women, the elderly, and the weak. In taking urine and finishing clothes, urine is easy to spill out of the container, causing hand or clothing pollution [4].

At present, most of the related research focuses on improving the testing instruments and the related personnel training. In contrast, the research on the collection process is relatively less. There are two types of designs related to urine collection in China [5,6]. One is a simpler and cheaper sampling cup improved based on the original sampling cup. The sampling cup's opening is usually funnel-shaped, and the lower part is connected to a urine cup through a catheter drainage; urine is thus collected through drainage. This kind of sampling cup partially solves the problem of urine splashing problems and inconvenient operations. However, its cost is higher than ordinary disposable plastic urine cups, so most hospitals do not widely use them. For example, the urine collection device produced by Qilu Hospital in Shandong, China, which uses sensors to identify whether the urine flows out or not, and controls the diversion by a magnet, can effectively intercept the interruption of urine. However, catheter cleaning is mainly through water flushing, which cannot completely ensure that urine is not contaminated. The other kind of design is to make sitting toilet intelligent and realize urine collection. By refitting the urine collection pipeline of the intelligent toilet, people can collect urine on the toilet. Alternatively, it is a collector made of sitting toilet shape; however, its cost is relatively high, and the equipment occupies a large area, so it is difficult to popularize in the squat toilet of most hospitals and public health examination centers [7-9].

Meanwhile, there are also many patents related to urine collection in other countries, even special collectors for women, children, and patients [10-13]. For example, the collection underwear with drainage tube and collector, collectors of various shapes, and intelligent toilet, of course, can simultaneously complete sampling and testing and get the results [14-17]. However, it is also difficult to promote in public places.

The collection system in this paper uses the STM32F103 microcontroller as the master controller [1820]. It uses an infrared remote controll to control the motor to change the position of the urine cup, and the structure entirely fits the shape characteristics of plastic urine cups. It designs an automatic and easy-to-use human urine collection system for hospitals' toilet environments and public physical examinations. It can maintain the physical examination environment of the public physical examination center to the greatest extent and it's easy to maintain. There is no need to buy extra special-shaped urine cups. Which can save cost, ensure the accuracy of the test results, and improve the inconvenience of the collection process.

\section{System structure design}

\subsection{Design requirements}

In order to ensure that the urine collection device can adapt to the toilet environment of hospitals and public health examination centers, the structural design of the collection system mainly considers the following factors: 
1) Without changing the original squatting toilet environment, it is easy to install, dismantle and maintain;

2) Apply all kinds of urine cups, ensuring practicality;

3) Simple operation, it is also suitable for the elderly and the people with movement difficulties;

4) Ensure that samples are not contaminated during the collection process, hands and clothes are not polluted by urine, and people can take away the samples after finishing their clothes.

\subsection{Structural composition}

The urine collection system comprises an infrared remote control, a collection vehicle, and an external pedal. The infrared remote control is set on the toilet wall or door, convenient for users to operate. It sends control signals through function buttons to control the collection vehicle [21-23]. The collection vehicle is used to carry the urine cup and receive the infrared remote control's signals to move forward and backward [24]. The external pedal is embedded in the collection vehicle to provide the mobile track. It needs to be placed on the squatting pan to carry the tested person during the collection process. The external pedal is embedded with a collection vehicle to provide a moving track for the vehicle and carry the subject during the acquisition process.

\subsubsection{Infrared remote control}

The infrared remote controller that controls the vehicle movement mainly includes an infrared transmitter and five control buttons, namely Forward, Backward, Emergency Stop, One-button Acquisition, and Call for Rescue. The program controls the motor in the collection vehicle to achieve one-button acquisition functions, manual adjustment forward and backward movements, emergency stop, and rescue call.

\subsubsection{Collection vehicle}

The collection vehicle is mainly used to carry the urine cup, control the urine cup movement and sampling automatically, and is the core component for realizing One-button acquisition. It is mainly composed of STM32F103 Microcontroller Unit (MCU), battery, infrared receiver, pressure sensor, metal ring, buzzer, four moving wheels, driving motor, and waterproof shell. Among them, the main chip, motor, and battery are all in the waterproof shell, and the infrared receiver is embedded in the shell to receive the infrared control signal and control the motor rotation and buzzer alarm. The front end of the shell is a metal ring for placing the urine cup. The diameter of the metal ring fits various commonly used urine cups, and the metal ring is connected by metal wires through the shell. A pressure sensor is arranged at the connection between the metal wires and the shell to judge the urine's pressure value. When the pressure signal is greater than the preset threshold, stop sampling to avoid urine overflow.

Figure 1 shows the overall structure of the collection vehicle.

\subsubsection{External pedal}

The external pedal is placed above the squatting pan, and Fig. 2 shows the structure. It can support the subject without changing the toilet structure so that the urine passes through the opening of the pit and flows into the urine cup, which is carried by the carrying vehicle.

It takes into account the practical needs of the device to be exposed to a wet environment for a long time. To avoid the corrosion of urine, the whole pedal is made of non-toxic, odorless, tasteless, low temperature resistant, acid and alkali resistant, and low water absorption HDPE. It is placed on the two sides of the squatting pan, and the overall size of the pedal is suitable for most squatting toilets. The 


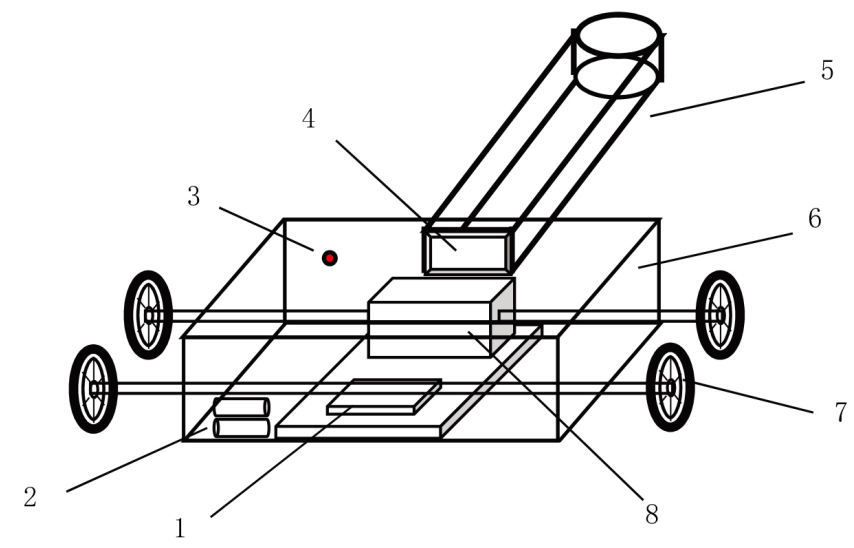

Fig. 1. Structure diagram of the collection vehicle.

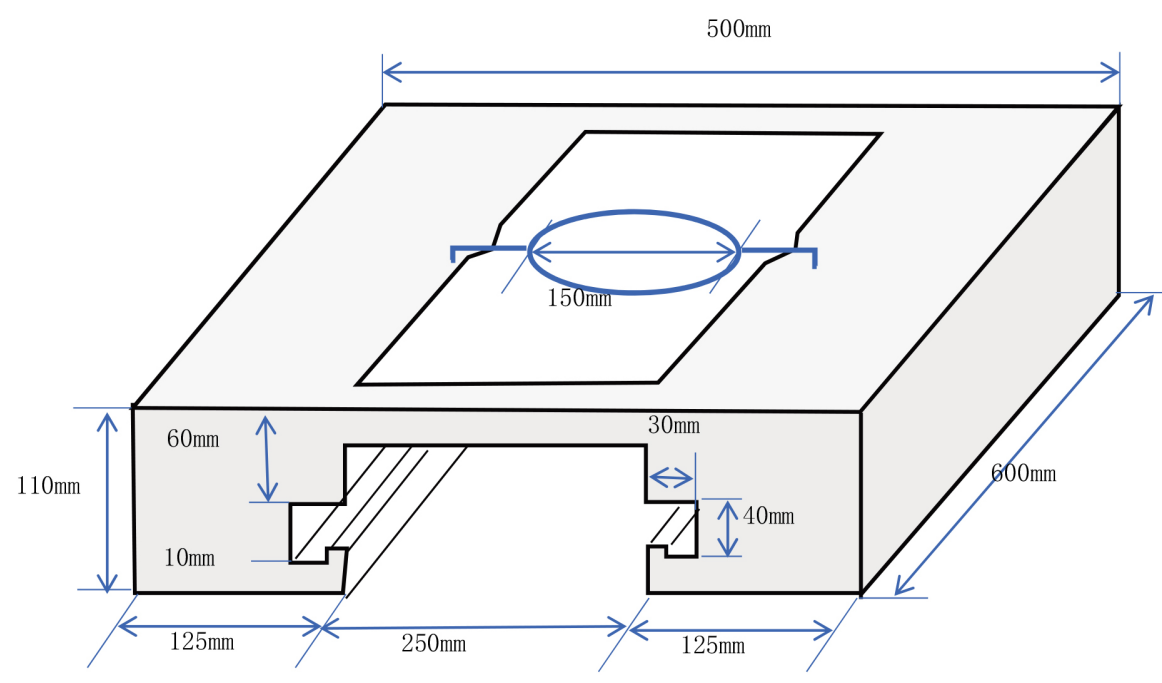

Fig. 2. Structure diagram of external pedal.

external pedal's top surface has a pit opening, and both sides are used as a tread surface to support the tested person. Also, a metal ring can be selectively placed on the top surface. The metal ring's diameter is larger than that on the collection vehicle, which can be used to control the position of ordinary disposable plastic urine cups and also can be used for funnel urine test cups. The pedal's bottom provides two support parts corresponding to the tread position, supported on both sides of the squatting pan. The pedal's inner sidewall has a moving track and a stop valve, whose width is the same as the wheel width of the collecting vehicle. The moving track plays a supporting role in collecting and carrying the vehicle, controlling the direction of movement and the fixed position.

\subsection{Workflow}

The whole system's workflow can be divided into four kinds: one-button acquisition, manual acquisition, emergency stop, and rescue call. 
First, open the power on the collection vehicle and manually adjust the collection vehicle's location to make the upper and lower metal rings align or adjust the initial position convenient to use the toilet. Then, place the urine cup and select the corresponding button according to the use situation.

One-button acquisition: one-button acquisition is suitable for average urine volume. Press the "Onebutton Acquisition" button of the infrared remote control. When the infrared receiving device receives the signal, the single-chip microcomputer controls the level and time delay through the built-in program to control the motor's positive and negative rotation and realize the forward and backward movement of the collection vehicle. The process is as follows: First, let the collection vehicle retreat for one second and then move forward for one second to return to the original position to get the midstream urine. Then, according to the pressure sensor's feedback value and comparing with the set threshold, when the urine weight is greater than the threshold, the collection vehicle retreats for one second. The tested person can put on his/her clothes before taking away the urine cup.

Manual collection: the manual collection is suitable for the case that the tested person's urine volume is not much, or the collection vehicle's initial position deviates. Through the "Forward" and "Backward" buttons of the infrared remote controller, one can manually control the collection vehicle to avoid collecting the forepart segment urine without polluting the hands.

Emergency Stop and Rescue Call: When particular circumstances occur during the acquisition process, press the "Emergency Stop" button to stop the acquisition vehicle's movement, and stop the one-button acquisition process. The audio alarm function can also be realized by the "Rescue Call" button when the body of the person under test is in emergencies.

\section{Software design}

The system uses the STM32F103 microcontroller with C language programming, according to the overall workflow, to determine the sequence of program operations. The main program calls the corresponding subroutines: infrared terminal decoding, motor forward driving, motor backward driving, motor constant driving, and delay subroutines.

According to the overall design, as Fig. 3 shows, the system's program flow is as follows. First, initialize the system and the infrared interruption. Then the interruption scan is performed. When the button on the remote control is pressed, it sends out an infrared signal, and the infrared signal arrives at the infrared receiving module to trigger an external interruption. It then enters the infrared terminal decoding subprogram to decode and store the infrared signal. Next, the microcontroller outputs the corresponding control signal to control the stepper motor to be positive, reverse, or stop. When the "Rescue Call" button is pressed, the buzzer is controlled to sound the alarm.

\section{System validation}

\subsection{Ethics statement}

The study was approved by "Medical Ethics Committee of Hebei Medical University" (2021003). All tested person provided written informed consent for the collection of the assessment of human automatic urine collection system and subsequent analysis.

\subsection{Subjects}

In the public physical examination center, open recruitment is conducted for people taking part in physical examination and medical personnel. 


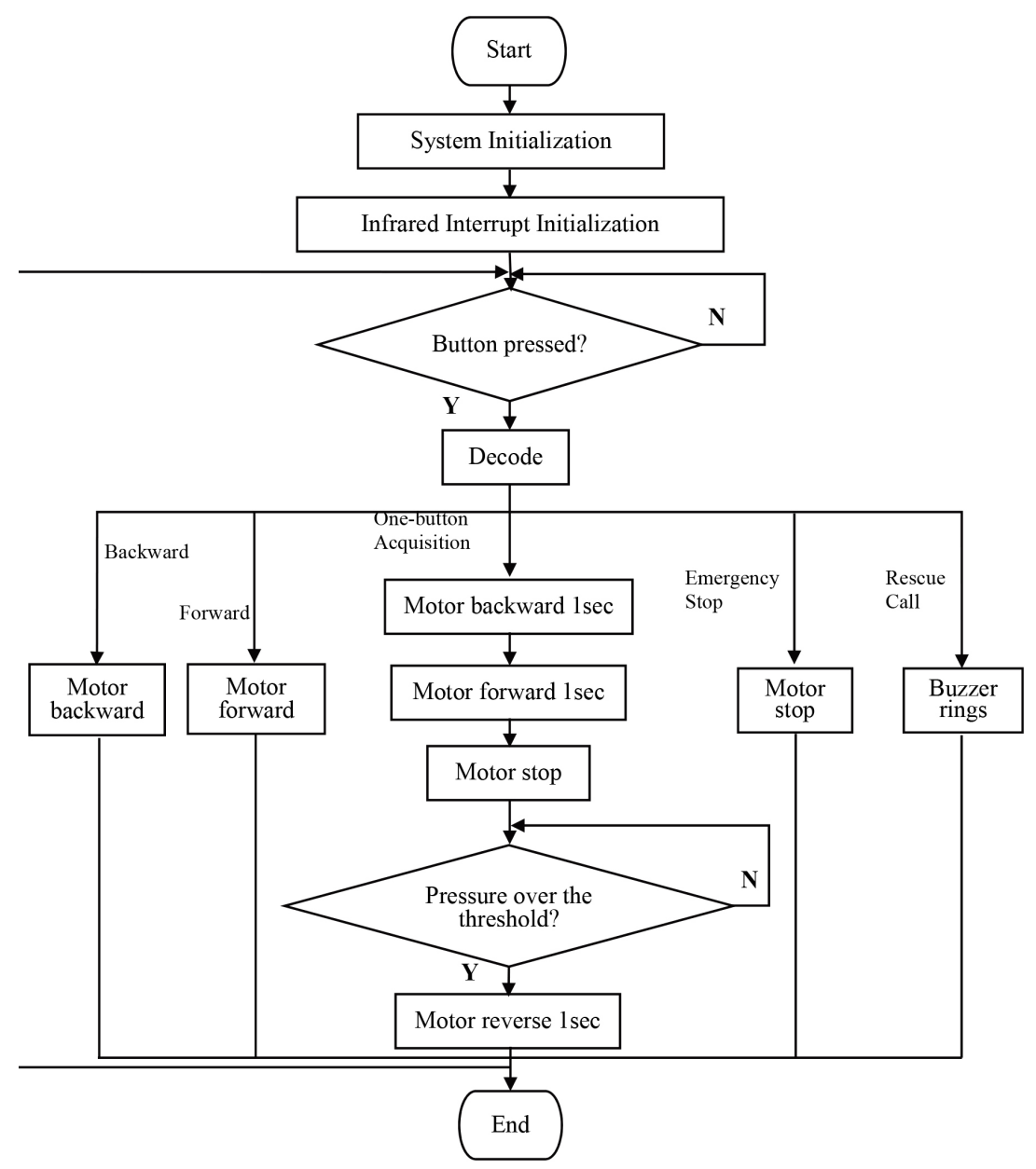

Fig. 3. Flow chart of system program.

Recruitment: from May 2020 to June 2021, volunteers who expressed interest in participating in the study received the study protocol in writing as well as verbal responses to any questions.

Inclusion criteria: (1) aged between 20 and 65, (2) no mental or neurological disorders, (3) normal or corrected-to-normal vision, (4) can use the toilet independently and urinate normally, (5) have no other major health problems.

Exclusion criteria: (1) older than 65 and younger than 20, (2) abnormal vision, (3) cannot use the toilet independently, (4) abnormal urination, (5) other midway abandonment.

Randomization: for subject allocation, subjects were randomly selected.

Sixty-two subjects participated in the study by public recruitment. They included 50 tested persons and 12 medical staff. The 12 medical personnel all work in the public physical examination center, including laboratory personnel, rehabilitation personnel, and management personnel of the physical examination center.

\subsection{Test procedure}

The sixty-two subjects should read the informed consent form carefully and sign the consent form 
Table 1

Comparison of scores between the two groups

\begin{tabular}{|c|c|c|c|c|c|c|c|c|c|c|c|}
\hline \multirow[t]{2}{*}{ No. } & \multicolumn{2}{|c|}{ Score } & \multirow[t]{2}{*}{ No. } & \multicolumn{2}{|c|}{ Score } & \multirow[t]{2}{*}{ No. } & \multicolumn{2}{|c|}{ Score } & \multirow[t]{2}{*}{ No. } & \multicolumn{2}{|c|}{ Score } \\
\hline & First & Second & & First & Second & & First & Second & & First & Second \\
\hline T1 & 75 & 54 & T17 & 58 & 6 & T33 & 76 & 55 & T49 & 72 & 57 \\
\hline $\mathrm{T} 2$ & 89 & 76 & T18 & 88 & 62 & T34 & 76 & 58 & T50 & 76 & 68 \\
\hline T3 & 72 & 62 & T19 & 62 & 50 & T35 & 82 & 72 & M1 & 88 & 65 \\
\hline T4 & 64 & 58 & T20 & 83 & 73 & T36 & 8 & 58 & M2 & 7 & 57 \\
\hline T5 & 7 & 6 & $\mathrm{~T} 21$ & 84 & 64 & T37 & 66 & 58 & M3 & 88 & 68 \\
\hline T6 & 82 & 59 & T22 & 73 & 70 & T38 & 84 & 62 & M4 & 62 & 50 \\
\hline $\mathrm{T} 7$ & 60 & 57 & T23 & 88 & 77 & T39 & 65 & 54 & M5 & 75 & 63 \\
\hline T8 & 60 & 65 & T24 & 62 & 52 & T40 & 73 & 63 & M6 & 84 & 64 \\
\hline T9 & 7 & 57 & T25 & 72 & 61 & T41 & 68 & 6 & M7 & 62 & 52 \\
\hline $\mathrm{T} 1$ & 62 & 50 & T26 & 85 & 65 & T42 & 84 & 64 & M8 & 72 & 61 \\
\hline $\mathrm{T} 11$ & 75 & 63 & T27 & 68 & 57 & T43 & 7 & 57 & M9 & 73 & 63 \\
\hline T12 & 70 & 62 & T28 & 75 & 67 & T44 & 62 & 50 & M1 & 76 & 58 \\
\hline T13 & 7 & 55 & T29 & 68 & 57 & T45 & 84 & 64 & M11 & 72 & 61 \\
\hline T14 & 66 & 54 & T3 & 73 & 62 & T46 & 7 & 58 & M12 & 66 & 6 \\
\hline T15 & 67 & 57 & T31 & 72 & 65 & T47 & 69 & 58 & & & \\
\hline T16 & 85 & 6 & T32 & 80 & 63 & T48 & 80 & 66 & & & \\
\hline
\end{tabular}

according to their own wishes. After that, they will be trained to use the human urine automatic collection system and collect urine twice by themselves. One time, the auxiliary system was used as the experimental group; Another time without auxiliary system, as a control group. Then, through the questionnaire survey, the two acquisition processes were scored. The content of the questionnaire includes (1) subject information: number, type (tested person or medical personnel), age, gender, and (2) a total of 100 points were scored in five aspects: 15 points for total collection time, 20 points for middle urine collection control, 20 points for urine splashing, 20 points for contaminated hands and clothes, and 25 points for convenient collection (for a tested person) or sample pollution (for medical personnel).

According to the score difference of each person scoring, the system trial experience is divided into four levels. Four levels for a tested person: (1) S1-S2 $\geqslant 20$, Very reasonable system design and the overall use process is convenient; (2) $10 \leqslant \mathrm{~S} 1-\mathrm{S} 2<20$, Reasonable system design, more convenient than direct sampling; (3) $0 \leqslant \mathrm{~S} 1-\mathrm{S} 2<10$, Reasonable system design, but troublesome to use; (4) S1-S2 $<0$, System design is not reasonable enough and troublesome to use. Four levels for medical personnel: (1) S1-S2 $\geqslant$ 20 , Sample pollution is small and meets the requirement of the midstream urine collection; It is suitable for popularization; (2) $10 \leqslant \mathrm{~S} 1-\mathrm{S} 2<20$, Sample pollution is small and the midstream urine sampling is basically consistent; It is slightly better than direct sampling; (3) $0 \leqslant \mathrm{~S} 1-\mathrm{S} 2<10$, Sample pollution is normal and midstream urine sampling is difficult to guarantee; No significant advantages; (4) S1-S2 $<0$, Sample pollution is large and midstream urine sampling is difficult to guarantee; It is better to sample directly.

Subsequently, Statistical Product and Service Solutions software was used to conduct paired sample mean $t$-test on the scores of the two groups, and the significance level was set as 0.05 .

\subsection{Results}

The scoring comparison of the two collections is shown in Table 1.

The results are distributed according to different levels, and the results are shown in Fig. 4. It showed that for the tested person, $96 \%$ of the respondents thought that the system design was reasonable, and $22 \%$ thought that the system was troublesome to use; for the medical personnel, $91.7 \%$ thought that the sampling system was in line with the sampling requirements, and the pollution was small. 
Table 2

Statistical analysis results of the two groups

\begin{tabular}{lcccc}
\hline & Average score & Standard deviation & $t$ & $P$ \\
\hline Experimental group & 73.30 & 8.33 & 13.937 & $0.000^{* *}$ \\
Control group & 60.72 & 6.16 & & \\
\hline
\end{tabular}

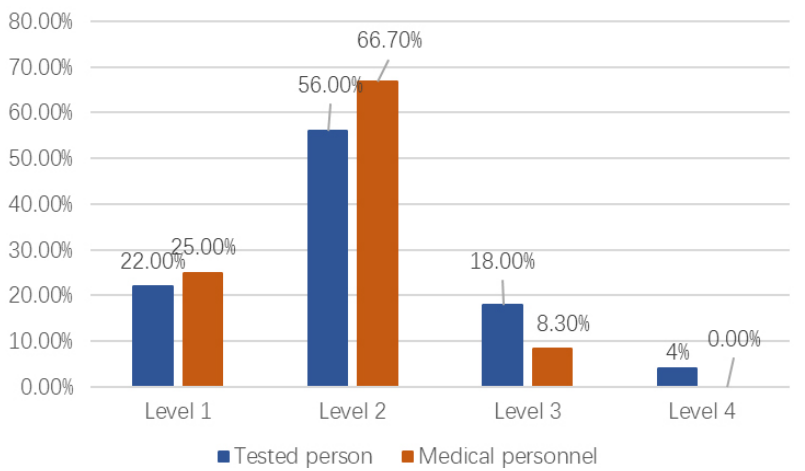

Fig. 4. Investigation results of human urine automatic collection system.

In addition, through the statistical analysis of the scores of the two groups, the results are shown in Table 2. Compared with the traditional acquisition mode, the average score of using the automatic acquisition system is higher. The paired sample mean test results show that the significance $\mathrm{P}$ is less than 0.05 , indicated a significant difference. It shows that the automatic acquisition system is better than the traditional acquisition method.

\section{Discussion}

This article reports an automatic urine collection system for automatic urine collection at hospitals at all levels and public health examination centers. Unlike the urine collection device produced by Qilu Hospital in Shandong, China, the collection device does not contain a catheter, so it is unnecessary to clean the catheter and avoid sample contamination. Compared with the intelligent toilet, it is slightly inconvenient to use, but it is also more suitable for hospitals and public health examination centers with a large number of tests [4-6].

In this article, a comparison is made between using the device and not using it. The statistical results showed that there was a significant difference between the two groups. Although it is not very targeted to the collector for special populations [10-13], it still has a high application value from the results. The device has low cost and good effect, and it can be popularized in public places compared with the intelligent toilet, which can complete sampling and testing simultaneously $[14,16]$.

Of course, because healthy people complete the testing process of the system, the safety and convenience of the special population need to be further tested. Although the evaluation is good, the objective evidence of whether the urine is not easy to be contaminated is still insufficient due to the subjective evaluation method. Therefore, the focus of the next step is to optimize the system further, improve the adaptability to the special population, and verify the pollution degree of the collected samples by using objective evidence. Before that, the test results can not be completely confirmed to be accurate, so the system is still in the experimental stage. 


\section{Conclusion}

The system uses the STM32F103 single-chip computer as the central controller. It uses an infrared remote control to control the motor to adjust the position of the urine cup, thus realizing the automatic collection of human urine. It can also be adjusted manually according to the use situation and has an alarm function. The overall size is based on the squatting pan, which fits the size and shape characteristics of various disposable plastic urine cups, even not affected by the replacement of urine cups. It can realize an automatic collection of midstream urine under normal conditions, the manual collection under particular circumstances, emergency stop, and rescue call. Through the trial survey, there was a significant difference between the statistical results of using the device and not using the device $(t=13.937, P=0.000)$. The trial evaluation is satisfactory and is suitable for hospitals at all levels and public health examination centers, where a large quantity of tests has to be performed, a shortage of medical personnel exists, or the process of urine collection cannot be strictly regulated. It can solve the test error caused by the nonstandard sampling and avoid embarrassment in a sampling operation. It has reasonable practicability and promotion value.

\section{Acknowledgments}

The authors thank all subjects who participated in the research and acknowledge the financial support from the Bureau of Science and Technology of Hebei Province Key Research and Development Program Project (Grant No. 182177170), and Hebei Provincial Foundation for the Introduction of Overseas Personnel (Grant No. C20210344).

\section{Conflict of interest}

The authors declare that they have no competing interests.

\section{References}

[1] Or H, Zvi B, Shai L, David L, Raphael G, Yehuda Z. Early non-invasive detection of breast cancer using exhaled breath and urine analysis. Computers in Biology and Medicine. May. 2018; 96: 227-232. doi: 10.1016/j.compbiomed.2018.04.002.

[2] Katica M, Ahmed NH, Salkić A, Mukača A, Bašić A, Dervševć E. The role of organic urine sediment analysis in the monitoring of renal diseases. Journal of Advances in VetBio Science and Techniques. Apr. 2019; 4(1): 40-50. doi: 10.31797/vetbio.524209.

[3] Sabaté Arroyo XA, Grases Freixedas F, Bauzà Quetglas JL, Guimerà Garcia J, Pieras Ayala E. Relationship of endoscopic lesions of the renal papilla with type of renal stone and $24 \mathrm{~h}$ urine analysis. BMC Urology. Apr. 2020; 20(46): 1-6. doi: 10.1186/s12894-020-00615-4.

[4] Chinegwundoh F. Urine sample collection: Issues and a solution. Trends in Urology \& Men's Health. Jan. 2018; 9(1): 16-18. doi: 10.1002/tre.615.

[5] Yang P, Chen Z, Yin L, Peng Y, Li X, Cao X, et al. Salt intake assessed by spot urine on physical examination in Hunan, China. Asia Pacific Journal of Clinical Nutrition. Dec. 2019; 28(4): 845-856. doi: 10.6133/apjen.201912_28(4).0022.

[6] Chen Y, Geng Y, Yao H, Yin D, Hu Y, Zhang Y, et al. Physical examination service utilization among seasonal immigrants in Sanya, China: a cross-sectional study. 2020. doi: 10.21203/rs.3.rs-15427/v1.

[7] Singh K, Palasanthiran P, Hamilton ST, Rawlinson WD. Cotton wool balls are an effective urine collection tool for CMV PCR for diagnosing congenital cytomegalovirus infection. Pathology. Jun. 2020; 52(4): 504-506. doi: 10.1016/j. pathol.2020.03.009. 
[8] Kingsberry L. Implementation of urine collection tubes with preservatives increases accuracy of urine test results and reduces number of CAUTIs reported. American Journal of Infection Control. Jun. 2019; 47(6): 40-41. doi: 10.1016/ j.ajic.2019.04.097.

[9] Nishi H, Yoshida Y, Inoue R, Takemura K, Nangaku M. Regional variance in the use of urine dipstick test for outpatients in Japan. Nephrology. Sep. 2020; 25(9): 676-682. doi: 10.1111/nep.13715.

[10] Parr BS, Mohrbacker JA. Urine collection system for women, U.S. Patent 10716 699, Jul. 21, 2020.

[11] VanMiddendorp EJ, Sims TS, Merritt AA, Keshavarzi PN, Dykstra MS, Kramer TD, et al. External female urine collection system and related method, U.S. Patent 10806 623, Oct. 20, 2020.

[12] Gonzalez AS, Flores AT, Reyes ADH, Mendez RG. Urine collection device for dependent patients, U.S. Patent 10034 659, Jul. 31, 2018.

[13] Kasirye A. Undergarment having urine-collection system, U.S. Patent 10624 778, Apr. 21, 2020.

[14] Gotbeter B. Device to facilitate urine specimen collection, U.S. Patent Application 14/880,459, Apr. 14, 2016.

[15] Kramer H, Wagschal H, Wagschal J. Urine sample collection apparatus, U.S. Patent 9974 520, May. 22, 2018.

[16] Nishtala V, Taylor WF, Hine RM. Multi-functional and modular urine collection system, U.S. Patent 9216 242, Dec. 22, 2015.

[17] Li B, Li Z, Yang C, Zheng S, Design of electronic lock based on single-chip microcomputer, in: Proc. 4th ICEEECS, 2016, Atlantis Press. Advances in Computer Science Research, vol. 50.

[18] Qin H. Elevator Drive Control system based on single Chip Microcomputer, in: Proc. 8th MCEI, 2018, Jun. Available: doi: 10.2991/mcei-18.2018.27.

[19] Yang F, Ma C, Li H. Design of wireless video transmission system based on STM32 microcontroller, in: Proc. AIP Conference Proceedings, May. 2017, AIP Publishing LLC, Vol. 1820, No. 1: 060010. doi: 10.1063/1.4977325.

[20] Han X, Hu E, Xing SU. Design and Implementation of an Industrial Intelligent Infrared Remote Controller, DEStech Transactions on Computer Science and Engineering cscme, 2019.

[21] Lin YW, B.vLin Y, Hsiao CY, Wang YY. IoTtalk-RC: Sensors as universal remote control for aftermarket home appliances. IEEE Internet of Things Journal. Jun. 2017; 4(4): 1104-1112. doi: 10.1109/JIOT.2017.2715859.

[22] Adiono T, Tandiawan B, Fuada S, Muttaqin R, Fathany MY, Adijarto W, et al. Prototyping design of IR remote controller for smart home applications, in: Proc. TENCON 2017-2017 IEEE Region 10 Conference, Penang, Malaysia, Nov. 2017, pp. 1304-1308.

[23] Satyasrikanth P, Penna M, Bolla DR. Automatic toll collection system using RFID. IJCSMC. Aug. 2016; 5(8): 247-225.

[24] Ma M, Huang B, Wang B, Chen J, Liao L. Development of an energy-efficient smart socket based on STM32F103. Applied Sciences. Nov. 2018; 8(11): 2276. doi: 10.3390/app8112276. 